\section{OPEN ACCESS}

Edited by:

Adolfo M. García, Universidad de San Andrés, Argentina

Reviewed by: Jian Wang,

Fudan University, China

Diana López-Barroso,

University of Malaga, Spain

*Correspondence: James B. Rowe james.rowe@mrc-cbu.cam.ac.uk

Received: 03 March 2021 Accepted: 28 June 2021 Published: 19 July 2021

Citation: Peterson KA, Jones PS, Patel N, Tsvetanov KA, Ingram R, Cappa SF, Lambon Ralph MA, Patterson K

Garrard $P$ and Rowe JB

(2021) Language Disorder in Progressive Supranuclear Palsy and Corticobasal Syndrome: Neural Correlates and Detection by the MLSE Screening Tool. Front. Aging Neurosci. 13:675739. doi: 10.3389/fnagi.2021.675739

\title{
Language Disorder in Progressive Supranuclear Palsy and Corticobasal Syndrome: Neural Correlates and Detection by the MLSE Screening Tool
}

\author{
Katie A. Peterson ${ }^{1}$, P. Simon Jones ${ }^{1}$, Nikil Patel ${ }^{2}$, Kamen A. Tsvetanov ${ }^{3}$, Ruth Ingram ${ }^{4}$, \\ Stefano F. Cappa ${ }^{5,6}$, Matthew A. Lambon Ralph ${ }^{7}$, Karalyn Patterson ${ }^{1,7}$, Peter Garrard ${ }^{2}$ \\ and James B. Rowe ${ }^{1,7 *}$
}

\begin{abstract}
${ }^{1}$ Department of Clinical Neurosciences and Cambridge University Hospitals NHS Trust, University of Cambridge, Cambridge, United Kingdom, ${ }^{2}$ Department of Neurosciences, St. George's, University of London, London, United Kingdom, ${ }^{3}$ Department of Psychology, University of Cambridge, Cambridge, United Kingdom, ${ }^{4}$ Division of Neuroscience and Experimental Psychology, University of Manchester, Manchester, United Kingdom, ${ }^{5}$ IUSS Cognitive Neuroscience Center (ICoN), University Institute for Advanced Studies IUSS, Pavia, Italy, ${ }^{6}$ Dementia Research Center, IRCCS Mondino Foundation, Pavia, Italy, ${ }^{7}$ MRC Cognition and Brain Sciences Unit, University of Cambridge, Cambridge, United Kingdom
\end{abstract}

Background: Progressive supranuclear palsy (PSP) and corticobasal syndrome (CBS) affect speech and language as well as motor functions. Clinical and neuropathological data indicate a close relationship between these two disorders and the non-fluent variant of primary progressive aphasia (nfvPPA). We use the recently developed Mini Linguistic State Examination tool (MLSE) to study speech and language disorders in patients with PSP, CBS, and nfvPPA, in combination with structural magnetic resonance imaging (MRl).

Methods: Fifty-one patients (PSP $N=13$, CBS $N=19$, nfvPPA $N=19$ ) and 30 age-matched controls completed the MLSE, the short form of the Boston Diagnostic Aphasia Examination (BDAE), and the Addenbrooke's Cognitive Examination III. Thirtyeight patients and all controls underwent structural MRI at 3 Tesla, with T1 and T2-weighted images processed by surface-based and subcortical segmentation within FreeSurfer 6.0.0 to extract cortical thickness and subcortical volumes. Morphometric differences were compared between groups and correlated with the severity of speech and language impairment.

Results: CBS and PSP patients showed impaired MLSE performance, compared to controls, with a similar language profile to nfvPPA, albeit less severe. All patient groups showed reduced cortical thickness in bilateral frontal regions and striatal volume. PSP and nfvPPA patients also showed reduced superior temporal cortical thickness, with additional thalamic and amygdalo-hippocampal volume reductions in nfvPPA. Multivariate analysis of brain-wide cortical thickness and subcortical volumes with MLSE domain scores revealed associations between performance on multiple speech and 
language domains with atrophy of left-lateralised fronto-temporal cortex, amygdala, hippocampus, putamen, and caudate.

Conclusions: The effect of PSP and CBS on speech and language overlaps with nfvPPA. These three disorders cause a common anatomical pattern of atrophy in the left frontotemporal language network and striatum. The MLSE is a short clinical screening tool that can identify the language disorder of PSP and CBS, facilitating clinical management and patient access to future clinical trials.

Keywords: progressive supranuclear palsy, corticobasal syndrome, speech, language, aphasia

\section{INTRODUCTION}

Progressive supranuclear palsy (PSP) and corticobasal degeneration are primary degenerative tauopathies affecting movement and cognition (Litvan et al., 1996; Armstrong et al., 2013; Burrell et al., 2014; Höglinger et al., 2017). Speech and language deficits are common in both disorders, but their recognition has been hampered by the lack of a brief but sensitive clinical assessment tool (Peterson et al., 2019). In this article, we use the Mini Linguistic State Examination (MLSE; Patel et al., 2020) to investigate the range of speech and language deficits in PSP and the corticobasal syndrome (CBS), and their neural correlates in structural magnetic resonance imaging (MRI).

The classical phenotype of PSP, Richardson's syndrome, is characterised by vertical supranuclear gaze palsy, axial rigidity, and postural instability, with cognitive impairment (Steele et al., 1964; Litvan et al., 1996). Richardson's syndrome is highly suggestive of PSP pathology, however, other common phenotypes have been described, including a presentation with speech and language deficits (PSP-SL; Respondek and Höglinger, 2016; Höglinger et al., 2017). The clinical syndrome of CBS is characterised by the combination of motor deficits (progressive asymmetrical akinetic rigidity, dystonia, tremor, myoclonus) and cortical features (alien limb, apraxia, cortical sensory change; Riley and Lang, 1988; Boeve et al., 2003), with heterogeneity in the clinical presentations and underlying pathology (Armstrong et al., 2013; Alexander et al., 2014). CBS is commonly accompanied by impaired speech and language (Burrell et al., 2014; Peterson et al., 2019). Indeed, a non-fluent agrammatic presentation of CBS (CBS-NAV) is recognised in consensus clinical diagnostic criteria (Armstrong et al., 2013). Speech and language deficits also develop commonly in PSP and CBS after motor presentations (Catricalà et al., 2019; Dodich et al., 2019; Peterson et al., 2019; Murley et al., 2020).

The speech and language changes of PSP and CBS have much in common with the non-fluent variant of a primary progressive aphasia (nfvPPA). Indeed, there is considerable overlap in the criteria for diagnosing nfvPPA, PSP-SL, and CBS-NAV (Peterson et al., 2019). There is agrammatism, anomia, circumlocution, and impaired syntactic comprehension in the context of preserved single-word comprehension and object knowledge in patients with PSP-SL and CBS-NAV (Armstrong et al., 2013; Höglinger et al., 2017; Dodich et al., 2019), and subtle deficits in verbal production and sentence comprehension in PSP/CBS (Dodich et al., 2019). However, few studies have directly compared speech and language in PSP/CBS with nfvPPA. Burrell et al. (2018) found aphasic deficits on formal testing in PSP patients that were comparable in frequency and severity to those of an nfvPPA group. However, the PSP group were recruited mainly from a cognitive disorders clinic and may have overrepresented cognitive phenotypic presentations.

Although the speech and language changes of PSP and CBS have similarities to nfvPPA, it does not necessitate a common aetiology. However, there are overlapping neuropathological features including neuronal, oligodendroglial, and astrocytic inclusions that are immunoreactive for tau with 4 microtubule binding repeats (4R; Grossman, 2010; Dickson et al., 2011; Spinelli et al., 2017). PSP and CBS clinical signs often follow the presentation of nfvPPA (Kertesz and McMonagle, 2010; SantosSantos et al., 2016; Cerami et al., 2017; Gazzina et al., 2019), or primary progressive apraxia of speech (Josephs et al., 2014). The clinical and pathological overlap of PSP, CBS, and nfvPPA underlies the concept of a continuous spectrum of $4 \mathrm{R}$-Tauopathy disorders (Kertesz et al., 2005; Dickson et al., 2011; Murley et al., 2020) that extends to the functional anatomy of their cognitive deficits.

Here, we test the hypothesis that the three disorders have a common associated structural impairment in relation to their common effects on speech and language. The functional anatomy of language impairment in PSP and CBS has been identified by fluorodeoxyglucose positron emission tomography (Dodich et al., 2019). Patients with language presentations of these two conditions have hypometabolism in areas typical of nfvPPA (left fronto-insular and superior medial frontal cortex), whereas patients without language impairment showed predominant right-hemispheric involvement. At the group level, the disorders differ: PSP is associated with atrophy and hypometabolism of midbrain, striatal, and frontal regions, bilaterally (Kaat et al., 2011; Niccolini and Politis, 2016; Murley et al., 2020); CBS is associated with asymmetric hypometabolism and atrophy of frontoparietal cortex and basal ganglia (Niccolini and Politis, 2016; Murley et al., 2020); and nfvPPA is associated with atrophy and hypometabolism of the left frontal perisylvian region, anterior insula, and frontal operculum (Nestor et al., 2003; Gorno-Tempini et al., 2004; Preiß et al., 2019).

The MLSE was recently developed as a screening tool to identify and categorise speech and language deficits in 
neurological disorders (Patel et al., 2020). We, therefore, used the MLSE to compare PSP, CBS, and nfvPPA. The speech and language symptoms of PSP and CBS have been difficult to assess and characterise in non-specialist settings because of the lack of a validated brief language screening test. The MLSE is accurate for the assessment of primary progressive aphasias (Patel et al., 2020) but its performance in other neurological disorders is yet to be assessed. We included patients with a range of PSP and CBS phenotypic presentations to reflect the range of cases presenting to cognitive and movement disorders clinics, noting that speech and language impairment occurs in PSP/CBS patients even in those who do not meet criteria for PSP-SL or CBS-NAV (Dodich et al., 2019).

This study had two key aims: (1) to use the MLSE to evaluate and compare linguistic impairment in PSP, CBS, and nfvPPA; and (2) to investigate brain structural correlates of speech and language deficits in PSP, CBS, and nfvPPA. We predicted that the language profile in PSP and CBS would resemble that seen in nfvPPA. We also predicted that performance on the MLSE would be associated with cortical atrophy in a left-lateralised language network, specifically, the inferior frontal cortex, associated with agrammatic and apraxic speech (Gorno-Tempini et al., 2004).

\section{MATERIALS AND METHODS}

\section{Ethics}

Ethical approval was obtained from the London-Chelsea Research Ethics Committee (REC reference: 16/LO/1735). The study was sponsored by St. George's, University of London. All participants provided written informed consent.

\section{Participants}

Fifty-one patients (PSP $N=13, \mathrm{CBS} N=19$, nfvPPA $N=19$ ) were recruited through specialist cognitive neurology clinics at Addenbrooke's Hospital, Cambridge $(N=33)$, St. George's Hospital, London $(N=13)$, and Manchester Royal Infirmary and its associated clinical providers $(N=5)$. Patients were included if they had a clinical diagnosis of PSP based on the 2017 Movement Disorder Society criteria (Höglinger et al., 2017), CBS based on the 2013 Armstrong et al. criteria (Armstrong et al., 2013), or nfvPPA based on the 2011 Gorno-Tempini criteria (Gorno-Tempini et al., 2011). Nine of the PSP patients had probable PSP-Richardson's syndrome; the other four included one each of probable PSP-progressive gait freezing (PSP-PGF), probable PSP-SL, possible PSP-ocular motor dysfunction (PSP$\mathrm{OM}$ ), and possible PSP. Eight CBS patients had probable CBS; the others had probable CBS-NAV $(N=6)$, possible CBS-NAV (1), probable CBS presenting as PSP syndrome (CBS-PSP) (1), and possible CBS (3). One nfvPPA patient declared a native language other than English but had been highly fluent in English since childhood and predominately used English in day-to-day life. Patients were excluded if they had advanced dementia and were unable to understand the purpose of the study or follow task instructions. As part of their clinical assessment, PSP and CBS patients completed the PSP Rating Scale (Golbe and OhmanStrickland, 2007). Medication information was also collected for these groups.
Thirty healthy controls were recruited. Inclusion criteria for the healthy control group were: the absence of a diagnosis of any pathological process causing a cognitive disorder and/or subjectively reported cognitive decline; age between 40 and 75 years; English as a first language; willing to participate in a study investigating language and dementia. Healthy controls were recruited through the National Institute for Health Research "Join Dementia Research" register ${ }^{1}$ in Cambridge and London, patients' relatives, and via local advertisement.

\section{Cognitive and Language Assessment}

Participants completed the Addenbrooke's Cognitive Examination-III (ACE-III; Hsieh et al., 2013), the short form of the Boston Diagnostic Aphasia Examination (BDAE; Goodglass et al., 2001), and the Mini Linguistic State Examination (MLSE; Patel et al., 2020). The short form of the BDAE, designed to assess language changes after focal brain damage (mainly stroke), was used to augment the MLSE assessment of language functions. A composite score was calculated from the following subtests of the short form of the BDAE, selected to overlap with the MLSE subtasks: single word repetition $(\max =5)$, basic word discrimination $(\max =16)$, sentence repetition $(\max =2)$, the Boston Naming Test short form $(\max =15)$, and basic oral word reading $(\max =15)$, giving a maximum $\mathrm{BDAE}$ composite score of 53 to compare with the MLSE total score. Assessment sessions were video and/or audio recorded for offline scoring and analysis.

The MLSE is a brief language assessment tool designed for the assessment of progressive aphasia. It contains subtests which span the principal linguistic domains affected by PPA, as used to apply the diagnostic criteria (Gorno-Tempini et al., 2011): confrontation naming, repetition, word and sentence comprehension, semantic association, reading, writing and a connected speech task. Error-based scoring provides domain scores corresponding to key linguistic domains (motor speech, knowledge of phonological structure, semantic knowledge, syntactic knowledge, and auditory-verbal working memory) as well as an overall total score out of 100, with lower scores indicating poorer performance. The MLSE has shown high interrater agreement and diagnostic accuracy for the classification of primary progressive aphasic syndromes (>90\% accuracy using random forest classification; Patel et al., 2020).

\section{Data Management}

Study data were collected and managed using the Research Electronic Data Capture tool, a secure, web-based software platform designed to support data capture for research studies, hosted at the University of Cambridge and at St. George's, University of London (Harris et al., 2009, 2019).

\section{Magnetic Resonance Imaging Protocol}

Thirty-eight (PSP $N=11$, CBS $N=14$, nfvPPA $N=13$ ) patients and all controls underwent structural MRI at 3 Tesla with a T1-weighted magnetisation-prepared rapid acquisition gradient echo (MPRAGE) and T2-weighted sequences. Twenty-seven

\footnotetext{
${ }^{1}$ http://www.joindementiaresearch.nihr.ac.uk
} 
patients (PSP $N=9$, CBS $N=9$, nfvPPA $N=9$ ) and 20 controls were scanned at the Wolfson Brain Imaging Centre, University of Cambridge on a Siemens Prisma 3T MRI (T1 Sagittal iPAT 2 parameters: $\mathrm{TR}=2,000 \mathrm{~ms}, \mathrm{TE}=2.93 \mathrm{~ms}, \mathrm{TA}=306 \mathrm{~s}$, in-plane resolution $=1.1 \times 1.1 \mathrm{~mm}$, slice thickness $=1.1 \mathrm{~mm}$, Inversion Time $=850 \mathrm{~ms}$, Flip Angle $=8^{\circ}$; T2 Sagittal iPAT 2 parameters: $\mathrm{TR}=3,200 \mathrm{~ms}, \mathrm{TE}=401 \mathrm{~ms}, \mathrm{TA}=283 \mathrm{~s}$, in-plane resolution $=1.1 \times 1.1 \mathrm{~mm}$, slice thickness $=1.1 \mathrm{~mm}$, Inversion Time $=850 \mathrm{~ms}$, Flip Angle $\left.=120^{\circ}\right)$. Eleven patients $($ PSP $N=2$, CBS $N=5, \operatorname{nfvPPA} N=4$ ) and 10 controls were scanned at the St. George's Hospital Radiology Department on a Philips Achieva 3T MRI (T1 Sagittal SENSE parameters: TR $=6,600-6,900 \mathrm{~ms}$, $\mathrm{TE}=3.0-3.2 \mathrm{~ms}$, in-plane resolution $=1.1 \times 1.1 \mathrm{~mm}$, slice thickness $=1.1 \mathrm{~mm}$, Inversion Time $=850 \mathrm{~ms}$, Flip Angle $=8^{\circ}$; T2 Sagittal SENSE parameters: TR $=2,200 \mathrm{~ms}$, TE $=243 \mathrm{~ms}$, in-plane resolution $=1.1 \times 1.1 \mathrm{~mm}$, slice thickness $=1.1 \mathrm{~mm}$, Inversion Time $=850 \mathrm{~ms}$, Flip Angle $=90^{\circ}$ ). Three patients were scanned at the Manchester Royal Infirmary site but were not included in the MRI analysis in part because no healthy volunteers were scanned at this site (i.e., unable to control for an effect of scanner difference). The remaining 10 patients either declined or were ineligible for MRI.

\section{Analysis}

Demographic, cognitive, and subcortical volumetric data were analysed using RStudio and $\mathrm{R}$ version 4.0.2. A chi-square test was conducted to investigate differences in sex distribution between groups. Since Levene's test showed that the variances for years of education and ACE-III score were not equal, Welch's ANOVA with Games-Howell post hoc test was used. Age, symptom duration, the BDAE composite score, MLSE total and MLSE domain scores, and volumes of thalamus, caudate, nucleus accumbens, and brainstem were not normally distributed. Therefore, the Kruskal-Wallis rank sum test was used, with post hoc pairwise comparisons by the Dunn test. Multiple testing correction was conducted using the BenjaminiHochberg method (Benjamini and Hochberg, 1995). A corrected value of $p<0.05$ following Benjamini-Hochberg correction was considered significant.

The T1- and T2-weighted images were processed using FreeSurfer 6.0.0 recon-all pipeline. The T2 volume was used to aid the definition of the pial surface. All images were reviewed to confirm accurate segmentation. Group differences in cortical thickness between each patient group and the control group were tested using a univariate analysis with the group as a factor. Sex and age were included as covariates of no interest. All tests were performed vertex-wise subject to clusterwise correction for multiple comparisons using FreeSurfer's mri_glmfit-sim for a permutation analysis with 10,000 randomisations and an initial uncorrected height threshold of $p<0.01$. Non-parametric permutations analysis adequately controls for false positives at this height threshold (Greve and Fischl, 2018).

We examined the relationship between language impairments and imaging metrics using univariate and multivariate statistics. The univariate approach tested the relationship between MLSE total score and cortical thickness with gender and age as covariates of no interest. Correlational analyses for cortical thickness were performed at each vertex subject to clusterwise correction for multiple comparisons using a permutation analysis with 10,000 randomisations and an initial uncorrected height threshold of $p<0.01$. Clusters surviving a two-sided corrected cluster threshold of $p<0.05$ were deemed significant. To investigate the link between MLSE and subcortical brain regions, left and right subcortical structure volumes from FreeSurfer were combined. Partial correlations were used to investigate associations between volumes of subcortical structures with MLSE total score, covarying estimated total intracranial volume, age, and sex.

To assess the multivariate relationship between MLSE domain scores and brain structures, we adopted a two-level procedure (Tsvetanov et al., 2018, 2019; Passamonti et al., 2019). First, canonical correlation analysis (Hotelling, 1936) identified the linear relationship between the two multivariate datasets, namely structural values (cortical thickness and subcortical volume) and MLSE domain scores, providing pairs of latent variables (Structure-LV and MLSE-LV). Each latent variable is a linear combination of the original variables, optimised to maximise the correlation between each pair. Here, dataset 1 consisted of structural values of cortical thickness and subcortical volume [67 subjects $\times 83$ nodes: 68 from the Desikan-Killiany Atlas and 15 (left and right: thalamus, caudate, putamen, pallidum, hippocampus, amygdala, and nucleus accumbens; and the brainstem) from the automatic subcortical segmentation atlas within FreeSurfer (Fischl et al., 2002)], and dataset 2 included MLSE domain scores (67 subjects $\times 5$ domains). Covariates of no interest included scanner site, gender, age, and total intracranial volume. Next, we tested whether the identified relationship between the cortical thickness and subcortical volume profile (Structure-LV) and MLSE-LV was differentially expressed by groups. We performed a second-level analysis using multiple linear regression with a robust fitting algorithm as implemented in the Matlab fitlm.m function. Independent variables included subjects' brain structure scores from first level canonical correlation analysis, group information (patient vs. control) and their interaction (Structure-LV $\times$ Group). The dependent variable was subjects' MLSE-LV scores from the first-level analysis in the corresponding canonical correlation analysis.

\section{RESULTS}

\section{Participant Demographics}

Fifty-one patients (PSP $N=13, \mathrm{CBS} N=19, \mathrm{nfvPPA} N=19$ ) and 30 controls completed the language assessment, as summarised in Table 1. The groups were similar in age and sex. The patient groups showed similar symptom duration $(F<1)$. There was a significant difference across the groups in years of education. Post hoc pairwise comparisons indicated that the control group had significantly more years of education than each patient group (all $p \leq 0.003$ ), but the patient groups did not differ from each other on this variable. There was a significant difference across the groups in ACE-III total score: higher in the control group than each patient group (all $p \leq 0.001$ ). In addition, the nfvPPA mean ACE-III total score was lower than PSP $(p=0.019)$. 
Thirty-eight patients were included in the MRI analysis protocol. We compared those included vs. not-included in the MRI analysis in terms of demographic and cognitive measures (Supplementary Table 1). The included patients had higher ACE-III, BDAE composite, and MLSE total scores compared to not-included patients, but the two groups did not differ in sex distribution, age, years of education, or symptom duration.

Five (26\%) CBS patients and six (46\%) PSP patients were taking dopaminergic medication. Using the Tomlinson et al. (2010) formula, levodopa equivalent daily dose value for the CBS patients was $193 \mathrm{mg}(\mathrm{SD}=88.6)$ and for the PSP patients was $345 \mathrm{mg}(\mathrm{SD}=254.1) .15 \%$ of PSP and $11 \%$ of CBS patients were taking benzodiazepines, $23 \%$ of PSP and $11 \%$ of CBS patients were taking opiates, $31 \%$ of PSP and $11 \%$ of CBS patients were taking selective serotonin reuptake inhibitors, $31 \%$ of PSP and $11 \%$ of CBS patients were taking other antidepressants.

\section{Language Scores}

Figure 1 presents group performance for MLSE domains. There were differences in scores between the groups for MLSE total score $\left(\chi_{(3)}^{2}=58.52\right.$, adjusted $\left.p<0.001\right)$, motor speech $\left(\chi_{(3)}^{2}=48.90\right.$, adjusted $\left.p<0.001\right)$, phonological structure $\left(\chi_{(3)}^{2}=47.56\right.$, adjusted $\left.p<0.001\right)$, semantic knowledge $\left(\chi_{(3)}^{2}=39.40\right.$, adjusted $\left.p<0.001\right)$, syntactic knowledge $\left(\chi_{(3)}^{2}=46.56\right.$, adjusted $\left.p<0.001\right)$, and auditory-verbal working memory $\left(\chi_{(3)}^{2}=8.16\right.$, adjusted $\left.p=0.043\right)$. Results of post hoc pairwise comparisons using the Dunn test with
Benjamini-Hochberg adjustment (Benjamini and Hochberg, 1995) are presented in Figure 1.

Each patient group performed worse than controls for MLSE total score and all MLSE subdomain scores apart from auditory-verbal working memory (after correction for multiple comparisons). The nfvPPA group scored lower than PSP and CBS groups on MLSE total score, with no significant difference between PSP and CBS. The PSP and CBS patients' MLSE subdomain scores were similar, while nfvPPA patients scored significantly lower than CBS patients for motor speech, PSP patients for semantic knowledge, and both PSP and CBS patients for phonological structure and syntactic knowledge.

There was a significant difference in BDAE composite score across the groups $\left(\chi_{(3)}^{2}=46.63\right.$, corrected $\left.p<0.001\right)$, with the control group scoring significantly higher than each patient group but with no significant differences between patient groups (see Table 1).

MLSE scores were converted to per cent scores to visualise the pattern of linguistic impairment across the groups. As shown in Figure 2, the pattern of impairment on MLSE subdomains is comparable across PSP, CBS and nfvPPA but with greater severity in the nfvPPA patients.

\section{Imaging Results}

There were differences in cortical thickness between controls and each patient group as shown in Figure 3A. All patient groups showed bilateral cortical thinning in medial and lateral frontal
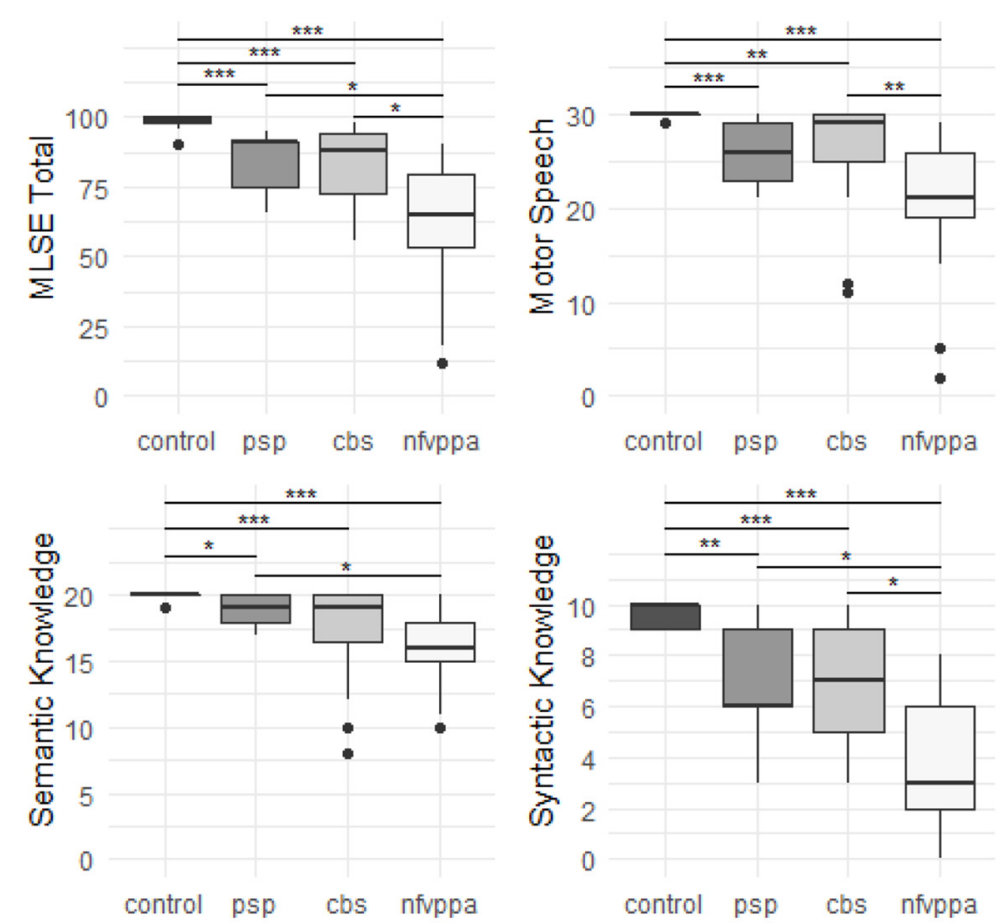
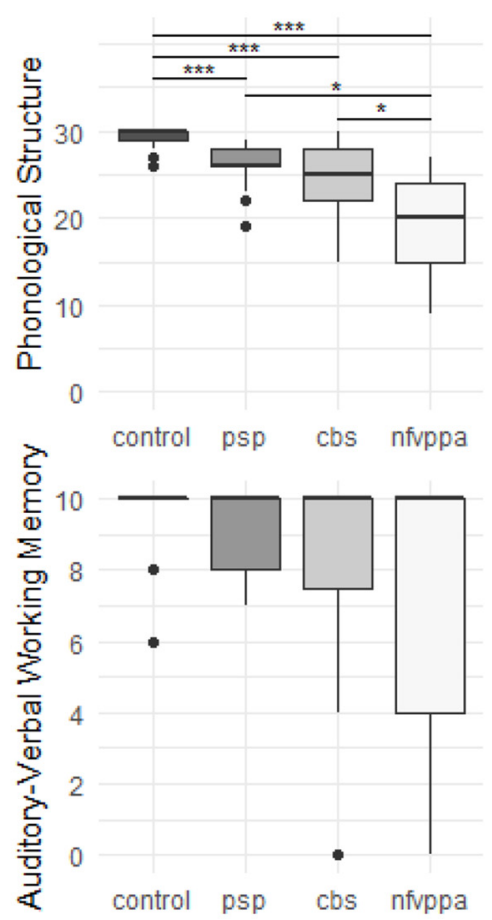

FIGURE 1 | Boxplots of group MLSE total and domain scores. The y-axes for each plot span the min to max scores. Significance markers represent adjusted $p$

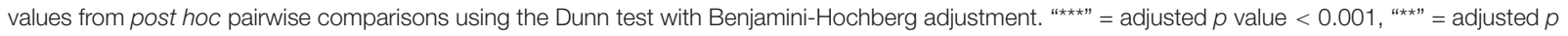
value $<0.01$, "*” = adjusted $p$ value $<0.05$. 
TABLE 1 | Demographic information for the study cohort.

\begin{tabular}{|c|c|c|c|c|c|}
\hline & Control & CBS & nfvPPA & PSP & Corrected $\mathbf{P}$ value \\
\hline $\mathrm{N}$ & 30 & 19 & 19 & 13 & - \\
\hline $\operatorname{Sex}(M / F)$ & $18 / 12$ & $11 / 8$ & $7 / 12$ & $8 / 5$ & 0.452 \\
\hline Age & 66.60 (4.33) & 70.37 (7.71) & 70.42 (6.95) & $69.23(6.03)$ & 0.063 \\
\hline \multicolumn{6}{|l|}{ Mean (SD) } \\
\hline $\begin{array}{l}\text { Education (years) } \\
\text { Mean (SD) }\end{array}$ & 15.97 (3.33) & $12.63(2.83)$ & $12.11(2.16)$ & $11.77(1.74)$ & $<0.001$ \\
\hline $\begin{array}{l}\text { Symptom duration (months) } \\
\text { Mean (SD) }\end{array}$ & NA & $49.47(45.15)$ & $35.63(30.46)$ & $45.62(26.94)$ & 0.590 \\
\hline $\begin{array}{l}\text { ACE-III score } \\
\text { Mean (SD) }\end{array}$ & $95.97(3.47)$ & $74.37(17.90)$ & $57.47(22.13)$ & $77.15(13.30)$ & $<0.001$ \\
\hline $\begin{array}{l}\text { BDAE composite } \\
\text { Mean (SD) }\end{array}$ & $52.68(0.50)$ & $47.42(5.88)$ & $39.29(12.51)$ & $46.58(6.93)$ & $<0.001$ \\
\hline $\begin{array}{l}\text { PSP Rating Scale } \\
\text { Mean (SD) }\end{array}$ & - & $29.28(18.17)$ & - & $36.50(16.15)$ & - \\
\hline
\end{tabular}

Note: corrected $P$ values are the result of Chi-squared, Welch's ANOVA, or Kruskal-Wallis rank sum tests for each row with Benjamini-Hochberg adjustment.
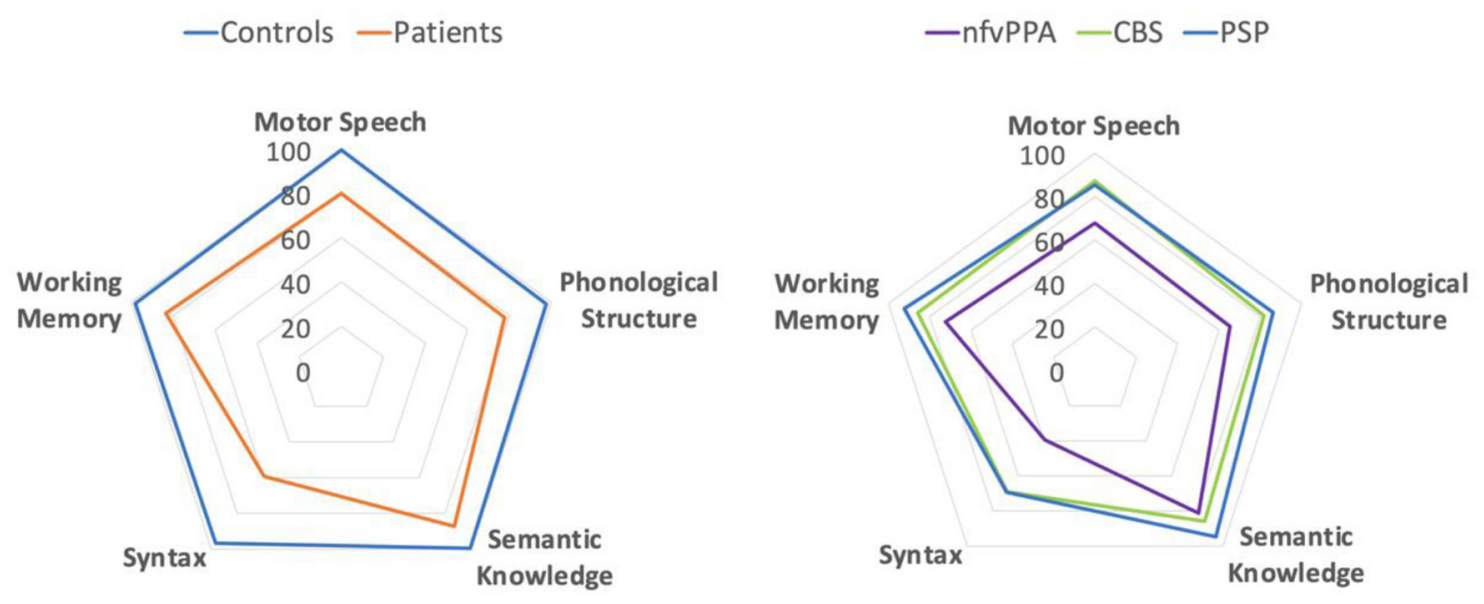

FIGURE 2 | Radar plots showing percentage scores for MLSE subdomains.
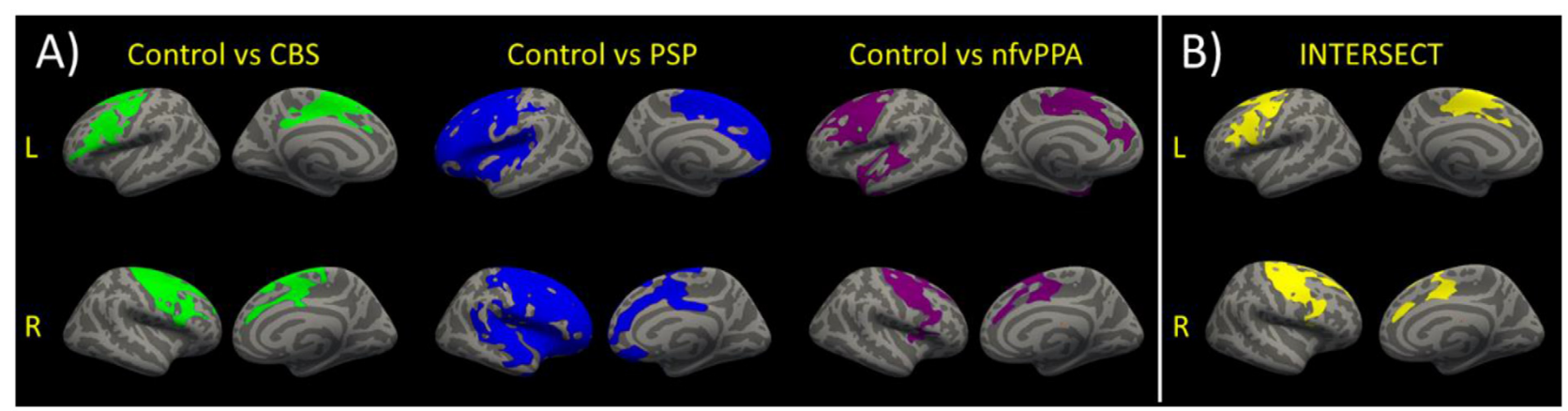

FIGURE 3 | (A) Reduced cortical thickness in each patient group vs. the control group. Clusters show regions of significant reductions in cortical thickness between each patient group and the controls following clusterwise correction at $p<0.05$. (B) For visualisation purposes, the intersect of the clusters for the three group comparisons of cortical thickness is shown. The intersect shows overlapping regions of reduced cortical thickness in the three patients vs. controls comparisons.

regions. The overlap between each patient-control cluster and Desikan-Killiany atlas regions is presented in Table 2. We created the intersect of the three patient-control analyses clusters to illustrate the region of commonality using the 'labels_intersect' command in FreeSurfer (Figure 3B). Overlapping regions of cortical thinning in the three patient groups encompassed 
inferior frontal, middle frontal, superior frontal, and precentral gyri (Figure 3B). The PSP and nfvPPA groups additionally showed cortical thinning in superior temporal regions, bilaterally in PSP and left-sided in nfvPPA. Correlational analysis between the MLSE total score and cortical thickness yielded no significant clusters following clusterwise correction.

There were group differences in subcortical volumes as shown in Table 3. All patient groups showed smaller putamen compared to controls. In addition, PSP patients showed reduced caudate volume compared to controls and reduced pallidum volume compared to all other groups; and nfvPPA patients showed reduced thalamus, caudate, and hippocampus volumes compared to controls and reduced amygdala volume compared to all other groups.

Partial correlations were conducted across the three patient groups to examine relationships between subcortical volumes and MLSE total score whilst controlling for estimated total intracranial volume, age and sex. There was a significant positive partial correlation between amygdala volume and MLSE total score $\left(r_{(33)}=0.377, N=38, p=0.026\right)$.

We assessed the multivariate relationship between MLSE domain scores and structural scores (cortical thickness and subcortical volumes) of 83 nodes across the brain using canonical correlation analysis. We identified one significant pair of latent variables (MLSE-LV and Structure-LV, $r=0.5731 p<0.001$; Figure 4). The Structure-LV expressed the highest loadings in the superior temporal cortex, prefrontal, inferior frontal and precentral regions, amygdala, hippocampus, putamen, and caudate, with a tendency for left lateralisation. The loading values for all structures are presented in Supplementary Table 2. The MLSE-LV expressed all domains, with the highest loadings on motor speech, phonology and syntax domains, followed by semantic and auditory-verbal working memory domains (Figure 4A). The positive loading values indicated that higher performance on MLSE domains (more so motor speech, phonology, and syntax domains) is associated with greater cortical thickness in the frontotemporal regions shown in Figure $4 \mathrm{~B}$ and in volumes of the amygdala, hippocampus, putamen and caudate (Figure 4C), with a tendency for left lateralisation. The remaining four components were not significant ( $p$ values $0.250,0.432,0.136,0.430$ for components $2-5$, respectively).

To test whether the observed relationship between MLSE-LV and Structure-LV is differentially expressed between controls and patients, we constructed a second-level regression model with robust error estimates by including Structure-LV subject scores, group information and their interaction term as independent variables and MLSE-LV as a dependent variable in addition to covariates of no interest (Figure 4D). We found evidence for a significant interaction $(r=0.562, p=0.006)$ indicating a stronger relationship between MLSE-LV and Structure-LV in the patients relative to controls.

\section{DISCUSSION}

This study reveals the severity and structural correlates of language impairment in PSP and CBS, using the Mini Linguistic
State Examination (MLSE). The PSP and CBS patients showed impaired performance on the MLSE domains of motor speech, phonological structure, semantic knowledge, and syntactic knowledge, but not auditory-verbal working memory. This pattern is similar to nfvPPA, consistent with previous reports of CBS-NAV or PSP-SL (Burrell et al., 2018; Catricalà et al., 2019; Dodich et al., 2019). PSP and CBS were similar to each other in the severity and range of language impairment.

We identified brain structural correlates of MLSE performance in PSP, CBS and nfvPPA. Multivariate analysis confirmed the association between a language component based on the MLSE domain scores (motor speech, phonology, and syntax loading most strongly) and a structural component (left-lateralised frontotemporal cortical thinning and subcortical atrophy). Further, we found cortical thinning common to all three patient groups in pre-frontal and precentral gyri. This accords with previous research showing that motor speech, phonology, and syntactic ability are the most affected linguistic domains in PSP, CBS, and nfvPPA (Burrell et al., 2018; Dodich et al., 2019; Peterson et al., 2019), and supports the sensitivity of MLSE to structural changes associated with language effects of PSP and CBS.

This atrophy is consistent with findings of hypometabolism in the left inferior frontal gyrus in patients with PSP-SL and CBS-NAV (Dodich et al., 2019), implicating this region in the emergence of a nfvPPA-type language profile. Despite this consistency, there is considerable heterogeneity in patterns of structural and functional impairment in these disorders. For example, some CBS patients show a pattern of language impairment resembling the logopenic variant of PPA, with impaired complex sentence repetition (Dodich et al., 2019), together with bilateral parietal hypometabolism, possibly reflecting underlying Alzheimer's disease pathology rather than corticobasal degeneration as the cause of CBS. Detailed analysis of linguistic impairment at the individual level in conjunction with pathological classification in PSP and CBS might provide more insight into the clinical-anatomical correlates of language impairment in these disorders.

The MLSE average assessment time was less than $20 \mathrm{~min}$, but this was sufficient to confirm mild to moderate impairment in motor speech, semantic knowledge, phonological abilities, and syntactic ability in PSP and CBS (Burrell et al., 2018; Peterson et al., 2019). This confirms the MLSE as a quick language screening tool for patients with mixed cognitive and movement disorders. Many language tests assume good visual and motor functions (e.g., tasks featuring visual stimuli or which require writing). Such tasks might disadvantage PSP patients due to their oculomotor abnormalities or disadvantage both PSP and CBS patients due to motor deficits. This complicates interpretation of results from investigations of language in these disorders because scoring is often binary, the reasons for task failures can be unclear and can differ across disorders (Peterson et al., 2019; Picillo et al., 2019). The MLSE addresses this longstanding issue by incorporating an error-based scoring system to capture language-specific contributions to impaired test performance, enabling one to tease apart linguistic deficits from one another and from other impairments. 
TABLE 2 | The overlap between each patient-control cortical thickness analysis cluster with Desikan-Killiany atlas regions shown in area mm² and percentage overlap.

\begin{tabular}{|c|c|c|c|c|}
\hline Structure Name & Hemisphere & $\begin{array}{l}\text { HC vs. CBS Cluster } \\
\left.\text { (Area } \mathrm{mm}^{2}, \%\right)\end{array}$ & $\begin{array}{l}\text { HC vs. PSP Cluster } \\
\text { (Area } \mathrm{mm}^{2}, \% \text { ) }\end{array}$ & $\begin{array}{l}\text { HC vs. nfvPPA Cluster } \\
\left.\text { (Area } \mathrm{mm}^{2}, \%\right)\end{array}$ \\
\hline \multicolumn{5}{|l|}{ Frontal } \\
\hline Superior Frontal & Left & $3305,50 \%$ & 6322, 95\% & $3803,57 \%$ \\
\hline \multirow{2}{*}{ Rostral Middle Frontal } & Left & $605,13 \%$ & $4549,95 \%$ & $1256,26 \%$ \\
\hline & Right & $1171,24 \%$ & $4840,98 \%$ & $365,7 \%$ \\
\hline Caudal Middle Frontal & Left & $1223,57 \%$ & $2072,97 \%$ & $1989,93 \%$ \\
\hline Pars Opercularis & Right & $618,48 \%$ & $1231,96 \%$ & $711,56 \%$ \\
\hline \multirow[t]{2}{*}{ Pars Triangularis } & Left & $216,19 \%$ & $1025,92 \%$ & 0 \\
\hline & Right & 0 & $805,64 \%$ & 0 \\
\hline \multirow[t]{2}{*}{ Pars Orbitalis } & Left & 0 & $259,47 \%$ & 0 \\
\hline & Right & 0 & $643,95 \%$ & 0 \\
\hline Lateral Orbitofrontal & Left & 0 & $1746,82 \%$ & 0 \\
\hline \multirow[t]{2}{*}{ Paracentral } & Left & $332,26 \%$ & $538,41 \%$ & $505,39 \%$ \\
\hline & Right & $503,33 \%$ & $287,19 \%$ & $169,11 \%$ \\
\hline \multirow[t]{2}{*}{ Frontal Pole } & Left & 0 & $201,100 \%$ & 0 \\
\hline & Right & 0 & $238,92 \%$ & 0 \\
\hline \multirow{2}{*}{ Rostral Anterior Cingulate } & Left & 0 & $350,51 \%$ & $245,36 \%$ \\
\hline & Right & 0 & $162,30 \%$ & 0 \\
\hline \multirow[t]{2}{*}{ Caudal Anterior Cingulate } & Left & $154,24 \%$ & $449,69 \%$ & $181,28 \%$ \\
\hline & Right & $49,7 \%$ & $185,26 \%$ & $52,7 \%$ \\
\hline \multicolumn{5}{|l|}{ Parietal } \\
\hline Superior Parietal & Left & 0 & 0 & 0 \\
\hline & Right & 0 & 0 & 0 \\
\hline Inferior Parietal & Left & 0 & 0 & 0 \\
\hline & Right & 0 & $289,6 \%$ & 0 \\
\hline Isthmus Cingulate & Left & $103,11 \%$ & 0 & 0 \\
\hline & Right & 0 & 0 & 0 \\
\hline Temporal & & & & \\
\hline Superior Temporal & Left & 0 & $1694,50 \%$ & $1910,56 \%$ \\
\hline & Right & 0 & $2422,76 \%$ & 0 \\
\hline Middle Temporal & Left & 0 & 0 & $216,8 \%$ \\
\hline & Right & 0 & $453,15 \%$ & 0 \\
\hline Inferior Temporal & Left & 0 & 0 & $600,22 \%$ \\
\hline & Right & 0 & $185,7 \%$ & 0 \\
\hline Banks of the Superior Temporal Sulcus & Left & 0 & 0 & $101,10 \%$ \\
\hline & Right & 0 & $565,64 \%$ & 0 \\
\hline Fusiform & Left & 0 & 0 & $469,17 \%$ \\
\hline & Right & 0 & 0 & 0 \\
\hline Transverse Temporal & Left & 0 & $433,100 \%$ & $280,65 \%$ \\
\hline & Right & 0 & $303,100 \%$ & 0 \\
\hline Entorhinal & Left & 0 & 0 & $224,48 \%$ \\
\hline & Right & 0 & 0 & 0 \\
\hline Temporal Pole & Left & 0 & 0 & $441,94 \%$ \\
\hline & Right & 0 & $232,52 \%$ & 0 \\
\hline Parahippocampal & Left & 0 & 0 & 0 \\
\hline & Right & 0 & 0 & 0 \\
\hline Insula & Left & 0 & $1715,85 \%$ & $294,15 \%$ \\
\hline & Right & 0 & $1606,87 \%$ & $229,12 \%$ \\
\hline
\end{tabular}


TABLE 2 | Continued

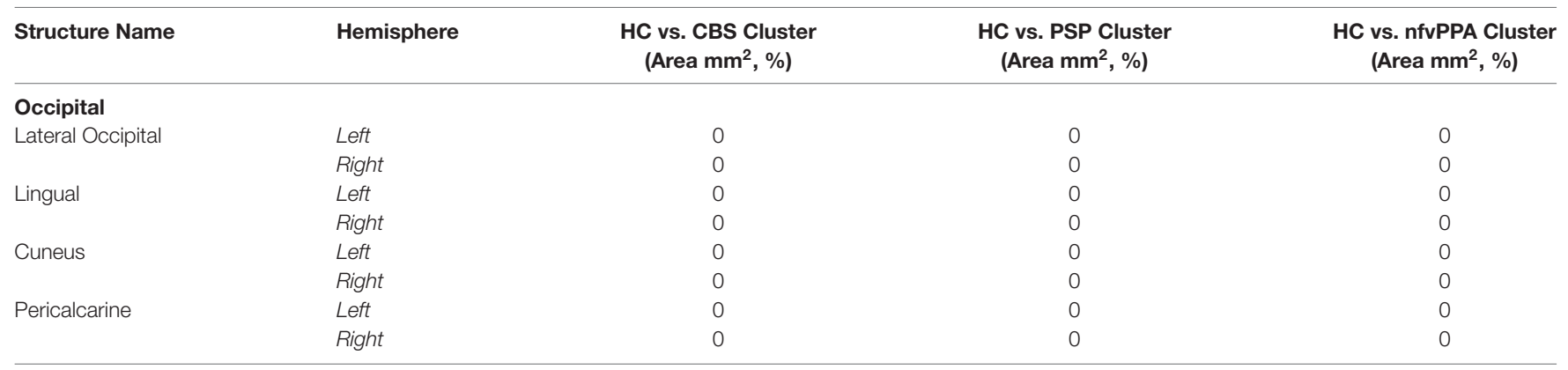

Note: only regions with $>5 \%$ overlap are shown.

TABLE 3 | Subcortical volumes.

\begin{tabular}{|c|c|c|c|c|}
\hline & Control Mean (SD) & PSP Mean (SD) & CBS Mean (SD) & nfvPPA Mean (SD) \\
\hline Thalamus & $13.33(1.47)$ & $12.73(3.84)$ & $12.06(1.69)$ & $11.74(1.51)^{*}$ \\
\hline Caudate & $6.85(1.08)$ & $6.01(1.27)^{*}$ & $6.39(1.15)$ & $5.66(0.82)^{\star}$ \\
\hline Putamen & $8.83(1.05)$ & $6.87(1.11)^{*}$ & $7.85(1.42)^{\star}$ & $7.21(1.00)^{\star}$ \\
\hline Hippocampus & $8.06(0.85)$ & $7.50(0.86)$ & $7.40(1.10)$ & $7.11(1.02)^{\star}$ \\
\hline Amygdala & $3.27(0.45)$ & $3.14(0.38)$ & $2.99(0.46)$ & $2.50(0.47)^{\star, \mathrm{a}, \mathrm{c}}$ \\
\hline Nucleus Accumbens & $0.96(0.13)$ & $0.90(0.35)$ & $0.86(0.18)$ & $0.82(0.17)$ \\
\hline
\end{tabular}

Note: volumes are presented in millilitres and left and right combined. Multiple testing correction was conducted using the Benjamini-Hochberg method (Benjamini and Hochberg, 1995). A corrected value of $p<0.05$ was considered significant. ${ }^{*}$ Significantly reduced vs. controls, a significantly reduced vs. CBS, ${ }^{b}$ significantly reduced vs. nfuPPA, ${ }^{c}$ significantly reduced vs. PSP.

A

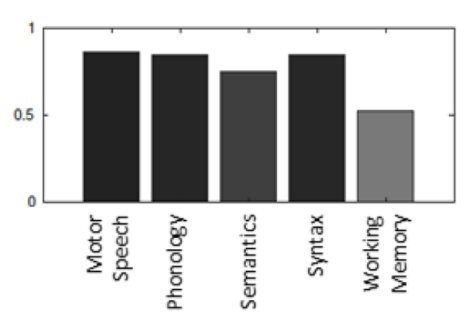

C

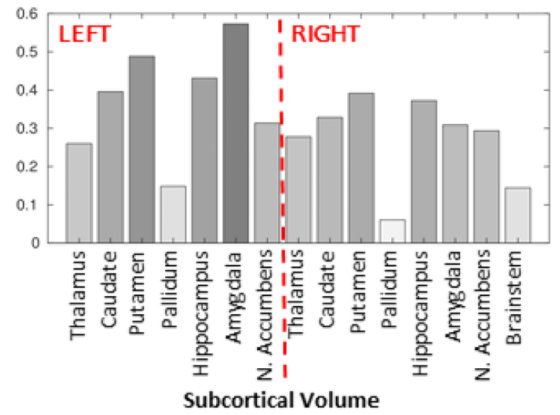

B
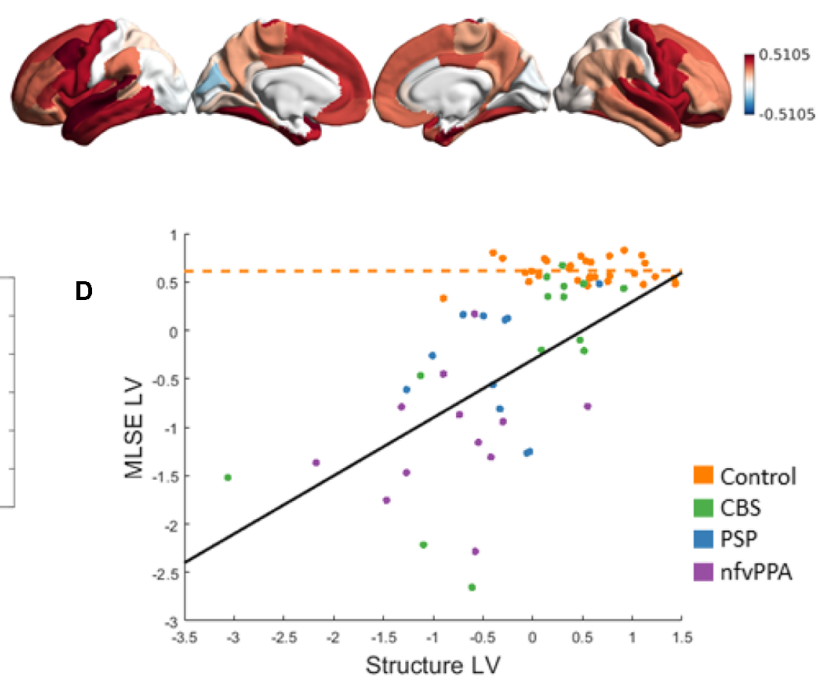

FIGURE 4 | (A) The MLSE latent variable (MLSE-LV) loadings. The MLSE-LV expressed all domains, with the highest loadings on motor speech, phonology and syntax, followed by semantic and auditory-verbal working memory domains. (B) and (C) The Structure latent variable (Structure-LV) loadings. The Structure-LV expressed high loadings in the superior temporal cortex, prefrontal, inferior frontal and precentral regions and in volumes of the amygdala, hippocampus, putamen and caudate, with a tendency for left lateralisation. (D) Visualisation of the interaction effect and the relationship between the MLSE-LV and the Structure-LV in the patients and controls. The relationship between MLSE-LV and Structure-LV was stronger in the patients relative to controls as visualised by black and orange trendlines, respectively, and confirmed formally by a significant interaction term $(r=0.562, p=0.006)$. Corticobasal syndrome (CBS), progressive supranuclear palsy (PSP) and nfvPPA groups are colour coded.

There are limitations to the present study. We do not have pathological validation in our sample although clinicopathological correlations of PSP are very high $(>90 \%$;
Gazzina et al., 2019). We have not examined phenotypic variance within groups other than language, due to the small group sizes and insufficient power but note that PSP and CBS can represent 
diverse phenotypes. The small group sizes reflect the rarity of these conditions and it may be due to type II error that we were unable to detect between-patient-group differences in the relationship between MLSE performance and brain structure. Future studies with larger group sizes are needed to robustly evaluate potential group-specific effects and to replicate our findings. The study did not aim to dissect phenotype-specific patterns of atrophy of linguistic impairment, but rather exploit cohort variance to examine structure-function relationships. We recognise a possible selection bias for the patients scanned, with more severely impaired patients less likely to have undergone MRI. This is supported by the poorer cognitive and language performance in the patients who were not included in the MRI analysis. Thus, our imaging results may be more reflective of early-to-mid stage PSP/CBS/nfvPPA. Finally, the crosssectional nature of this study precludes an interpretation of the progression of language profiles.

In conclusion, we find evidence for mild to moderate speech and language deficits in PSP and CBS which are similar in profile to nfvPPA. We have identified a shared anatomical substrate that correlates with linguistic impairment across these disorders, sensitive to MLSE profiling, consistent with the overlapping clinical and pathological spectrum of PSP, CBS, and nfvPPA.

\section{DATA AVAILABILITY STATEMENT}

We invite requests for the anonymised data for academic (noncommercialised) purposes, to the corresponding author. Data sharing may be subject to restrictions on some data types to protect confidentiality. Requests to access the datasets should be directed to james.rowe@mrc-cbu.cam.ac.uk.

\section{ETHICS STATEMENT}

The study was reviewed and approved by the London-Chelsea Research Ethics Committee (REC reference: 16/LO/1735).

\section{REFERENCES}

Alexander, S. K., Rittman, T., Xuereb, J. H., Bak, T. H., Hodges, J. R., and Rowe, J. B. (2014). Validation of the new consensus criteria for the diagnosis of corticobasal degeneration. J. Neurol. Neurosurg. Psychiatry 85, 923-927. doi: 10.1136/jnnp-2013-307035

Armstrong, M. J., Litvan, I., Lang, A. E., Bak, T. H., Bhatia, K. P., Borroni, B., et al. (2013). Criteria for the diagnosis of corticobasal degeneration. Neurology 80, 496-503. doi: 10.1212/WNL.0b013e31827f0fd1

Benjamini, Y., and Hochberg, Y. (1995). Controlling the false discovery rate: a practical and powerful approach to multiple testing. J. Royal Stat. Soc.: Series B (Methodological) 57, 289-300. doi: 10.1111/j.2517-6161.1995. tb02031.x

Boeve, B. F., Lang, A. E., and Litvan, I. (2003). Corticobasal degeneration and its relationship to progressive supranuclear palsy and frontotemporal dementia. Ann. Neurol. 54, S15-S19. doi: 10.1002/ana.10570

Burrell, J. R., Ballard, K. J., Halliday, G. M., and Hodges, J. R. (2018). Aphasia in progressive supranuclear palsy: as severe as progressive non-fluent aphasia. J. Alzheimer's Dis. 61, 705-715. doi: 10.3233/JAD170743

Burrell, J. R., Hodges, J. R., and Rowe, J. B. (2014). Cognition in corticobasal syndrome and progressive supranuclear palsy: a review. Mov. Disord. 29, 684-693. doi: $10.1002 / \mathrm{mds} .25872$

\section{AUTHOR CONTRIBUTIONS}

KAP, NP, RI, SC, ML, KP, PG and JR contributed to conception and design of the study. KAP, NP and RI collected the data. KAP, PJ and KT performed the statistical analysis. SC, ML, KP, PG and JR supervised the project. KAP wrote the first draft of the manuscript. KT wrote sections of the manuscript. All authors contributed to the article and approved the submitted version.

\section{FUNDING}

The work was supported by the Medical Research Council (MR/N025881/1; SUAG051/101400; SUAG048/101400), the Guarantors of Brain (101149), the Cambridge Centre for Parkinson-plus and NIHR Cambridge Biomedical Research Centre (BRC-1215-20014). The views expressed are those of the authors and not necessarily those of the NIHR or the Department of Health and Social Care.

\section{ACKNOWLEDGEMENTS}

We thank the NIHR Join Dementia Research service for support provided during recruitment.

\section{SUPPLEMENTARY MATERIAL}

The Supplementary Material for this article can be found online at: https://www.frontiersin.org/articles/10.3389/fnagi. 2021.675739/full\#supplementary-material.

SUPPLEMENTARY TABLE 1 | Comparison between patients who were or were not included in the MRI analysis.

SUPPLEMENTARY TABLE 2 | The Structure-LV loading values of each structure (from the Desikan-Killiany atlas) and subcortical volumes, arranged from highest to lowest.

Catricalà, E., Boschi, V., Cuoco, S., Galiano, F., Picillo, M., Gobbi, E., et al. (2019). The language profile of progressive supranuclear palsy. Cortex 115, 294-308. doi: 10.1016/j.cortex.2019.02.013

Cerami, C., Dodich, A., Greco, L., Iannaccone, S., Magnani, G., Marcone, A., et al. (2017). The role of single-subject brain metabolic patterns in the early differential diagnosis of primary progressive aphasias and in prediction of progression to dementia. J. Alzheimer's Dis. 55, 183-197. doi: 10.3233/JAD160682

Dickson, D. W., Kouri, N., Murray, M. E., and Josephs, K. A. (2011). Neuropathology of frontotemporal lobar degeneration-Tau (FTLDTau). J. Mol. Neurosci. 45, 384-389. doi: 10.1007/s12031-011 $-9589-0$

Dodich, A., Cerami, C., Inguscio, E., Iannaccone, S., Magnani, G., Marcone, A., et al. (2019). The clinico-metabolic correlates of language impairment in corticobasal syndrome and progressive supranuclear palsy. NeuroImage Clin. 24:102009. doi: 10.1016/j.nicl.2019. 102009

Fischl, B., Salat, D. H., Busa, E., Albert, M., Dieterich, M., Haselgrove, C., et al. (2002). Whole brain segmentation: automated labeling of neuroanatomical structures in the human brain. Neuron 33, 341-355. doi: 10.1016/s08966273(02)00569-x

Gazzina, S., Respondek, G., Compta, Y., Allinson, K. S. J., Spillantini, M. G., Molina-Porcel, L., et al. (2019). Neuropathological validation of the MDS-PSP 
criteria with PSP and other frontotemporal lobar degeneration. bioRxiv [Preprint]. doi: 10.1101/520510

Golbe, L. I., and Ohman-Strickland, P. A. (2007). A clinical rating scale for progressive supranuclear palsy. Brain 130, 1552-1565. doi: 10.1093/brain/awm032

Goodglass, H., Kaplan, E., and Barresi, B. (2001). Boston Diagnostic Aphasia Examination Third Edition (BDAE-3). Philadelphia, PA: Lippincott Williams and Wilkins.

Gorno-Tempini, M. L., Dronkers, N. F., Rankin, K. P., Ogar, J. M., Phengrasamy, L., Rosen, H. J., et al. (2004). Cognition and anatomy in three variants of primary progressive aphasia. Ann. Neurol. 55, 335-346. doi: 10.1002/ana.10825

Gorno-Tempini, M. L., Hillis, A. E., Weintraub, S., Kertesz, A., Mendez, M., Cappa, S. F., et al. (2011). Classification of primary progressive aphasia and its variants. Neurology 76, 1006-1014. doi: 10.1212/WNL.0b013e31821 $103 \mathrm{e} 6$

Greve, D. N., and Fischl, B. (2018). False positive rates in surface-based anatomical analysis. NeuroImage 171, 6-14. doi: 10.1016/j.neuroimage.2017.12.072

Grossman, M. (2010). Primary progressive aphasia: clinicopathological correlations. Nat. Rev. Neurol. 6, 88-97. doi: 10.1038/nrneurol.2009.216

Harris, P. A., Taylor, R., Minor, B. L., Elliott, V., Fernandez, M., O’Neal, L., et al. (2019). The REDCap consortium: building an international community of software platform partners. J. Biomed. Inform. 95:103208. doi: 10.1016/j.jbi. 2019.103208

Harris, P. A., Taylor, R., Thielke, R., Payne, J., Gonzalez, N., and Conde, J. G. (2009). Research electronic data capture (REDCap)-A metadata-driven methodology and workflow process for providing translational research informatics support. J. Biomed. Inform. 42, 377-381. doi: 10.1016/j.jbi.2008. 08.010

Höglinger, G. U., Respondek, G., Stamelou, M., Kurz, C., Josephs, K. A., Lang, A. E., et al. (2017). Clinical diagnosis of progressive supranuclear palsy: the movement disorder society criteria. Mov. Disord. 32, 853-864. doi: $10.1002 / \mathrm{mds} .26987$

Hotelling, H. (1936). Relations between two sets of variates. Biometrika 28, 321-377. doi: 10.2307/2333955

Hsieh, S., Schubert, S., Hoon, C., Mioshi, E., and Hodges, J. R. (2013). Validation of the addenbrooke's cognitive examination III in frontotemporal dementia and Alzheimer's disease. Dement. Geriatr. Cogn. Disord. 36, 242-250. doi: 10.1159/000351671

Josephs, K. A., Duffy, J. R., Strand, E. A., Machulda, M. M., Senjem, M. L., Gunter, J. L., et al. (2014). The evolution of primary progressive apraxia of speech. Brain 137, 2783-2795. doi: 10.1093/brain/awu223

Kaat, L. D., Chiu, W. Z., Boon, A. J. W., and van Swieten, J. C. (2011). Recent advances in progressive supranuclear palsy: a review. Curr. Alzheimer Res. 8, 295-302. doi: 10.2174/156720511795563809

Kertesz, A., and McMonagle, P. (2010). Behavior and cognition in corticobasal degeneration and progressive supranuclear palsy. J. Neurol. Sci. 289, 138-143. doi: 10.1016/j.jns.2009.08.036

Kertesz, A., McMonagle, P., Blair, M., Davidson, W., and Munoz, D. G. (2005). The evolution and pathology of frontotemporal dementia. Brain 128, 1996-2005. doi: 10.1093/brain/awh598

Litvan, I., Agid, Y., Calne, D., Campbell, G., Dubois, B., Duvoisin, R. C., et al. (1996). Clinical research criteria for the diagnosis of progressive supranuclear palsy (Steele-Richardson-Olszewski syndrome): report of the NINDS-SPSP International workshop. Neurology 47, 1-9. doi: 10.1212/wnl.47.1.1

Murley, A. G., Coyle-Gilchrist, I., Rouse, M. A., Jones, P. S., Li, W., Wiggins, J., et al. (2020). Redefining the multidimensional clinical phenotypes of frontotemporal lobar degeneration syndromes. Brain 143, 1555-1571. doi: 10.1093/brain/awaa097

Nestor, P. J., Graham, N. L., Fryer, T. D., Williams, G. B., Patterson, K., and Hodges, J. R. (2003). Progressive non-fluent aphasia is associated with hypometabolism centered on the left anterior insula. Brain 126, 2406-2418. doi: 10.1093/brain/awg240
Niccolini, F., and Politis, M. (2016). A systematic review of lessons learned from PET molecular imaging research in atypical parkinsonism. Eur. J. Nucl. Med. Mol. Imaging 43, 2244-2254. doi: 10.1007/s00259-016-3464-8

Passamonti, L., Tsvetanov, K. A., Jones, P. S., Bevan-Jones, W. R., Arnold, R., Borchert, R. J., et al. (2019). Neuroinflammation and functional connectivity in alzheimer's disease: interactive influences on cognitive performance. J. Neurosci. 39, 7218-7226. doi: 10.1523/JNEUROSCI.2574-18.2019

Patel, N., Peterson, K. A., Ingram, R., Storey, I., Cappa, S. F., Catricala, E., et al. (2020). The Mini linguistic state examination (MLSE): a brief but accurate assessment tool for classifying primary progressive aphasias. MedRxiv [Preprint]. doi: 10.1101/2020.06.02.20119974

Peterson, K. A., Patterson, K., and Rowe, J. B. (2019). Language impairment in progressive supranuclear palsy and corticobasal syndrome. J. Neurol. 268, 796-809. doi: 10.1007/s00415-019-09463-1

Picillo, M., Cuoco, S., Carotenuto, I., Abate, F., Erro, R., Volpe, G., et al. (2019). Clinical use of SAND battery to evaluate language in patients with progressive supranuclear palsy. PLoS One 14:e0223621. doi: 10.1371/journal.pone. 0223621

Preiß, D., Billette, O. V., Schneider, A., Spotorno, N., and Nestor, P. J. (2019). The atrophy pattern in Alzheimer-related PPA is more widespread than that of the frontotemporal lobar degeneration associated variants. NeuroImage: Clin. 24:101994. doi: 10.1016/j.nicl.2019.101994

Respondek, G., and Höglinger, G. U. (2016). The phenotypic spectrum of progressive supranuclear palsy. Parkinsonism Relat. Disord. 22, S34-S36. doi: 10.1016/j.parkreldis.2015.09.041

Riley, D. E., and Lang, A. E. (1988). Corticobasal ganglionic degeneration (CBGD): further observations in six additional cases [abstract]. Neurology 38:360.

Santos-Santos, M. A., Mandelli, M. L., Binney, R. J., Ogar, J., Wilson, S. M., Henry, M. L., et al. (2016). Features of patients with nonfluent/agrammatic primary progressive aphasia with underlying progressive supranuclear palsy pathology or corticobasal degeneration. JAMA Neurol. 73, 733-742. doi: 10.1016/j.ajoc.2021.101124

Spinelli, E. G., Mandelli, M. L., Miller, Z. A., Santos-Santos, M. A., Wilson, S. M., Agosta, F., et al. (2017). Typical and atypical pathology in primary progressive aphasia variants. Ann. Neurol. 81, 430-443. doi: 10.1002/ana.24885

Steele, J. C., Richardson, J. C., and Olszewski, J. (1964). Progressive supranuclear palsy. a heterogeneous degeneration involving the brain stem, basal ganglia and cerebellum with vertical gaze and pseudobulbar palsy, nuchal dystonia and dementia. Arch. Neurol. 10, 333-359. doi: 10.1001/archneur.1964. 00460160003001

Tomlinson, C. L., Stowe, R., Patel, S., Rick, C., Gray, R., and Clarke, C. E. (2010). Systematic review of levodopa dose equivalency reporting in Parkinson's disease. Mov. Disord. 25, 2649-2685. doi: 10.1002/mds.23429

Tsvetanov, K. A., Henson, R. N., Jones, P. S., Mutsaerts, H.-J., Fuhrmann, D., Tyler, L. K., et al. (2019). The effects of age on resting-state BOLD signal variability is explained by cardiovascular and neurovascular factors. bioRxiv [Preprint]. 836619. doi: 10.1111/psyp.13714

Tsvetanov, K. A., Ye, Z., Hughes, L., Samu, D., Treder, M. S., Wolpe, N., et al. (2018). Activity and connectivity differences underlying inhibitory control across the adult life span. J. Neurosci. 38, 7887-7900. doi: 10.1523/JNEUROSCI. 2919-17.2018

Conflict of Interest: The authors declare that the research was conducted in the absence of any commercial or financial relationships that could be construed as a potential conflict of interest.

Copyright (C) 2021 Peterson, Jones, Patel, Tsvetanov, Ingram, Cappa, Lambon Ralph, Patterson, Garrard and Rowe. This is an open-access article distributed under the terms of the Creative Commons Attribution License (CC BY). The use, distribution or reproduction in other forums is permitted, provided the original author(s) and the copyright owner(s) are credited and that the original publication in this journal is cited, in accordance with accepted academic practice. No use, distribution or reproduction is permitted which does not comply with these terms. 